\title{
Prevalence of heterophoria and its association with near fusional vergence ranges and refractive errors
}

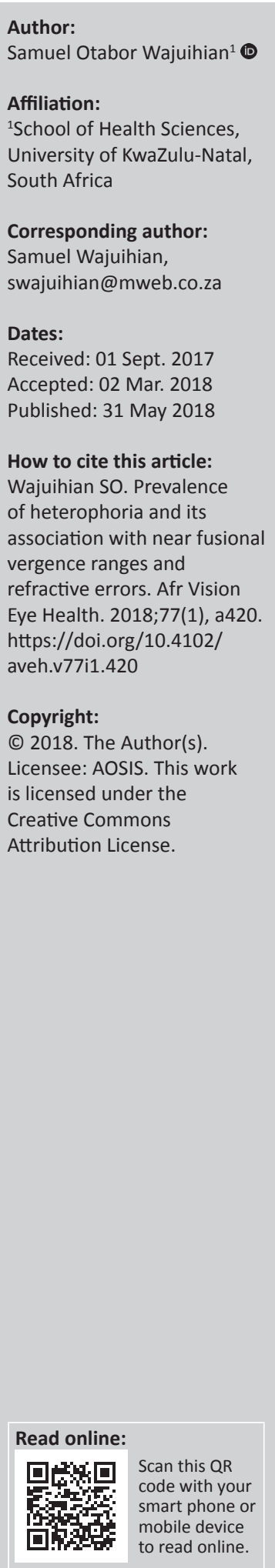

Background: Heterophoria is an error of binocular alignment that manifests only during monocular viewing or in conditions that disrupt binocular vision. Heterophoria is compensated for by fusional vergence through a mechanism that involves both sensory and motor fusion. The distribution of heterophoria has not been studied extensively in schoolchildren in South Africa and studies quantifying the magnitude of association between heterophoria and fusional vergences are scarce.

Purpose: The aim of this study was to determine the prevalence of heterophoria and investigate its associations with fusional vergences and refractive errors.

Methods: This was a cross-sectional study design and comprised data from 1056 high school students aged 13-18 years, who were randomly selected from 13 out of a sample frame of 60 high schools in uMhlathuze municipality in the province of KwaZulu-Natal, South Africa. The sample comprised 403 (38\%) males and 653 (62\%) females. The participants' mean age was $15.89 \pm 1.58$ years. Visual acuity, refractive errors, heterophoria and fusional vergences were evaluated using conventional optometric techniques.

Results: The overall prevalence of orthophoria at distance was 80.1\% (95\% confidence interval [CI]: $77.7 \%-82.6 \%$ ), that of exophoria was $13.9 \%$ (CI: $11.9 \%-16.0 \%$ ), while that of esophoria was $6.0 \%$ (CI: $4.5 \%-7.4 \%)$. At near, the prevalence estimates were as follows: exophoria, 542 (51.3\%, CI: $48.3 \%-54.2 \%$ ); orthophoria, 36.6\% (CI: 33.9\% - 39.5\%); esophoria, $12.1 \%$ (CI: $10.2 \%-14.0 \%$ ). On the severities of near phoria, the frequencies were as follows: mild (1-7 prism dioptres [pd]), $41 \%$ (CI: 38.3\% - 44.1\%); moderate (8-13 pd), 9.7\% (CI: $7.8 \%-11.5 \%)$; and severe (> $13 \mathrm{pd}), 1.1 \%$ (CI: $0.6 \%-1.8 \%)$. For the severities of fusional vergences, the distributions were as follows: positive fusional vergences, mild (15-22 pd), 88.3\% (CI: 86.4\% - 90.4\%); moderate ( 7 to $<15$ pd), $10.1 \%$ (CI: $8.2 \%-11.9 \%)$; and severe ( $\leq 7 \mathrm{pd}), 1.6 \%$ (CI: $0.9 \%-2.4 \%)$. For negative fusional vergences, the percentage of anomalous cases ( $\geq 22 \mathrm{pd}$ ) was $14.2 \%$ (CI: $12.3 \%-16.3 \%$ ), while that for normal $(<22)$ was $85.8 \%$ (CI: $83.7 \%-87.7 \%)$. For vertical phoria, the prevalence was $2.7 \%$ and $3.6 \%$ at near. There was a weak but significant inverse correlation between near exophoria and positive fusional vergence break $(r=-0.10, p=0.01)$, whereas exophoria and negative fusional vergence break were positively correlated $(r=0.14, p=0.01)$. Esophoria was inversely correlated with negative fusional vergence break values $(r=0.13, p=0.01)$ and was positively correlated with positive fusional vergence break $(r=0.13, p=0.03)$. Chi-square tests showed that exophoria was associated with astigmatism $(p=0.01)$, while esophoria was significantly associated with myopia $(p=0.01)$, astigmatism $(p=0.01)$ and anisometropia $(p=0.01)$.

Conclusion: The data for far and near distance, horizontal and vertical heterophoria were not normally distributed. Orthophoria was most prevalent at far, exophoria at near and there was a significant association between phoria and fusional vergence. The significant associations between phoria and fusional vergence were at lower levels of fusional vergences.

\section{Introduction}

Heterophoria (abbreviated as 'phoria') is the relative deviation of the visual axes when the eyes are dissociated. ${ }^{1,2}$ Heterophoria may be classified as horizontal (esodeviation - visual axes converge, or exodeviation - visual axes diverge), according to the direction of the visual axes when the eyes are dissociated. ${ }^{1,2}$ In vertical deviations, one visual axis deviates above or below the other, relative to the reference eye, ${ }^{1,2}$ while a condition of oblique disorientation is referred to as 'cyclophoria' or 'torsional deviation'.1,2 Orthophoria occurs when the lines of sight cross at the object of regard. ${ }^{1,2}$ Normal binocular vision requires accurate alignment of the eyes, as 
well as sensory and motor components of fusion. ${ }^{1}$ Fusional (disparity) vergence is the vergence stimulated by retinal disparity that is aimed at maintaining single binocular vision. ${ }^{1,2}$ Dissociating the eyes removes the disparity cue used by fusional vergences to compensate for the misalignment and causes a shift in the appropriate phoria position. ${ }^{3,4}$

A phoria may be caused by high uncorrected refractive errors, accommodation-vergence anomalies and anatomical factors, as well as increased near-task demands. ${ }^{1,2}$ Heterophoria, fusional vergences and refractive errors are important clinical measures. Heterophoria is a measure of horizontal and vertical stress on the oculomotor system ${ }^{5}$ and may not result in symptoms. However, a phoria may become decompensated (manifest symptoms) when fusional vergence is inadequate to compensate for the demand. ${ }^{2,5}$ The symptoms in decompensated phoria include headaches, photophobia and eye strain and may affect visual efficiency and/or lowered academic performance in schoolchildren., ${ }^{2,5}$ Furthermore, both decompensated and uncompensated phoria could result in suppression or strabismus. ${ }^{2,5}$ Fusional convergence cannot be achieved if the eyes deviate excessively, even if the necessary retinal disparity control mechanisms exist in the brain. ${ }^{5}$ In addition, phoria and fusional vergence are variables applied to classify vergence anomalies and a guideline to predict if a patient may be symptomatic. ${ }^{6,7}$

Studies have investigated the distribution of heterophoria ${ }^{3,}$ $4,8,9,10,11,12,13,14,15,16,17,18,19,20,21,22,23,24,25,26,27,28,29,30,31,32,33,34,35,36,37,38,39,40,41,42$ and associations with fusional vergences $3,4,8,9,10,11,12,13,14,15,16,17,18,19,20,21,22$ $, 23,24,25,26,27,28,29,30,31,32,33,34,35,36$ and refractive errors $37,38,39,40,41,42$ in various populations. Consistent findings across the studies show that data from heterophoria are not normally distributed; orthophoria is the dominant phoria state at distance while exophoria dominates at near fixation. In addition, some degree of heterophoria occurs in $70 \%-80 \%$ of the population. ${ }^{3,4,8,9,10,11,12,13,14,15,16,17,18,19,20,21,22,23,24,25,26,27,28,29,30,31,32,33,34,35,36}$ Four available studies ${ }^{10,11,12,13}$ reported on the distribution of heterophoria in African populations. Mathebula et al. ${ }^{10}$ studied heterophoria in children aged 6-13 years in Limpopo and reported a mean of $2.5 \pm 2.37$ prism dioptre $(\mathrm{pd})$ exophoria for horizontal phoria at near fixation. In another prospective study, Mathebula ${ }^{11}$ reported data for 139 clinic patients whose ages ranged between 20 and 36 years. The mean horizontal phoria at near was $2.1 \pm 6.2$ pd exophoria. Makgaba $^{12}$ retrospectively analysed record cards of 336 patients (aged from 18 to 30 years) who were examined at the optometry clinic of the University of Limpopo. The nearhorizontal phoria was $3.84 \pm 4.80 \mathrm{pd}$ exophoria. Akpe et al. ${ }^{13}$ studied children aged between 5 and 19 years in Nigeria and found the prevalence of heterophoria was $23 \%$ at distance and $53.6 \%$ at near. However, heterophoria and its association with fusional vergences and refractive errors have not been studied in South African populations. In addition, there is little or no data specifically for high school children, a level in schooling that represents a critical stage of learning where there is increasing near demand compared to primary school.
This may give some unique information on the development of phoria and fusional vergences in school-going children.

Given the clinical importance of the associations among heterophoria, fusional vergences and refractive errors, especially regarding the prediction of symptoms, it is relevant to investigate the magnitude or severity of the demand and the fusional vergence ranges. Such data could enhance differential diagnosis. Therefore, the aim of this study was to determine the distribution of heterophoria and its association with fusional vergence ranges and refractive errors in a sample of schoolchildren. In contrast to previous studies, here, heterophoria was classified according to severity and investigated in relation to various levels of near-fusional vergences: its associations with refractive errors were also explored.

\section{Methods Study design}

This report is part of a cross-sectional study designed to quantify near-vision anomalies (refractive and accommodativevergence) and their association with symptoms in order to develop strategies on how to identify and treat them. The focus of this study is on near-vision measures in children aged 13-18 years.

\section{Participants and study setting}

The target population was black high school students in uMhlathuze municipality, and the study sample comprised students selected from 13 high schools out of a total sample frame of 60 high schools in this municipality of the province of KwaZulu-Natal, South Africa. Participants were selected using stratified, multistage cluster, random sampling (from the municipality to classroom levels). Students of African descent and of either gender were eligible to participate in the study. From the case history, participants were excluded if they had any systemic conditions such as diabetes, hypertension or multiple sclerosis and were on any systemic medication. Also excluded were children with amblyopia, suppression, strabismus, ocular diseases or nystagmus.

The study design, including the sampling and sample size calculation and details of testing procedures, are described in previous publications; ${ }^{43,44}$ details are omitted here as the techniques are conventional optometric procedures. ${ }^{6}$

\section{Materials and procedure}

The school principals provided the venues where the visual examinations were conducted. The purpose and procedure for every technique was carefully explained to each participant prior to the eye screening. To ensure that the participants understood all the instructions, trial testing was performed for complex tests such as accommodative facility and fusional vergences. The test procedures and testing conditions were standardised and an average of three test 
measurements were taken for near tests. The focus of this study was on near-vision functions; therefore, far-distance variables are not described in detail.

\section{Screening procedures}

Preliminary tests performed included visual acuity measurements and ocular health status assessment; suppression was evaluated at near using the Worth four-dot test and stereoacuity using the Randot Stereotest. Ocular motility was evaluated using the broad H-test. Refractive errors were determined objectively (without cycloplegia) with an autorefractor (Huvitz MRK-3100, Seoul, South Korea) and subjectively using the phoropter. Heterophoria was evaluated over the participants' subjective refraction and was evaluated using the von Graefe prism dissociation method after strabismus had been ruled out using the unilateral cover test. The fusional ranges were evaluated using a trial frame and prism bars. Negative fusional vergence (NFV) was routinely measured first, before positive fusional vergences (PFV), as the convergence responses stimulated during the base-out measurements may produce the adaptation of tonic vergence (related to the physiological position of rest and because of the extraocular muscles' tonus), which may bias the subsequent base-in values. . $^{6,711}$

\section{Data analysis}

All data were analysed by a statistician using the Statistical Package for Social Sciences (SPSS) version 21. Descriptive statistics were presented as means, standard deviations and medians. The Kolmogorov-Smirnov (K-S) test was used to test for normality of data. Chi-square tests and logistic regression models were performed to test for differences in proportions for categorical variables among groups. The Kruskal-Wallis equality-of-population rank non-parametric test was applied to compare differences in means for related measures among groups, and independent $t$-tests were used to compare the means of gender, age groups and grade levels. The non-parametric Spearman's rho correlation test was used to determine the strength of correlations between heterophoria and fusional vergences, while the chi-square test was applied to determine associations between phoria and refractive errors. The distributions of variables were presented using tables, graphs and proportions, and corresponding $95 \%$ confidence intervals were presented as an estimate of the prevalence.

The classification criteria (Table 1) for heterophoria ${ }^{45}$ and fusional vergences followed the criteria used in other studies, ${ }^{43,45}$ while vertical phoria followed the recommendation by Von Noorden. ${ }^{1}$ Another criteria considered was the Sheard's criterion proposes that for an individual to be comfortable (without experiencing symptoms), the fusional reserve opposing the heterophoria should be at least twice the size of the heterophoria: base out (PFV) for exophoria and base in (negative fusional vergence) for esophoria. ${ }^{6,7}$ Thus, failing the Sheard's criterion suggests that a patient may
TABLE 1: Classification criteria for heterophoria and fusional vergences according to magnitude.

\begin{tabular}{|c|c|}
\hline Clinical measures & Criteria \\
\hline \multicolumn{2}{|l|}{ Refractive error $(D)$} \\
\hline - Myopia & $\leq-0.50 \mathrm{D}$ \\
\hline - Hyperopia & $\geq+0.50 \mathrm{D}$ \\
\hline - Astigmatism & $\leq-0.75 \mathrm{D}$ \\
\hline - Anisometropia & $\begin{array}{l}\text { At least }-0.75 \mathrm{D} \text { difference between both eyes } \\
\text { spherical equivalent (sphere }+1 / 2 \text { cylinder) }\end{array}$ \\
\hline \multicolumn{2}{|l|}{ Heterophoria (pd) } \\
\hline \multicolumn{2}{|l|}{ Exophoria } \\
\hline - Orthophoria & 0 \\
\hline - Mild & $1-7$ \\
\hline - Moderate & $8-13$ \\
\hline - Severe & $>13$ \\
\hline \multicolumn{2}{|l|}{ Esophoria } \\
\hline - Orthophoria & 0 \\
\hline - Normal & $1-2$ \\
\hline - Anomalous & $>2$ \\
\hline \multicolumn{2}{|l|}{ Vertical phoria } \\
\hline \multicolumn{2}{|l|}{ Right hyperphoria } \\
\hline - Normal & $\leq 0.50$ \\
\hline - Anomalous & $>0.50$ \\
\hline \multicolumn{2}{|c|}{ Fusional vergence break (pd) } \\
\hline \multicolumn{2}{|l|}{ Positive } \\
\hline - Mild & $15-22$ \\
\hline - Moderate & $>7$ to $<15$ \\
\hline - Severe & $\leq 7$ \\
\hline \multicolumn{2}{|l|}{ Negative } \\
\hline - Normal & $\geq 22$ \\
\hline - Anomalous & $<22$ \\
\hline
\end{tabular}

Source: Wajuihian and Hansraj ${ }^{43}$ and Bade et al. ${ }^{45}$

D, dioptres; pd, prism dioptres.

be symptomatic. ${ }^{6,746}$ The fusion reserve ratio was calculated as the fusional convergence reserve divided by the prism value from the phoria test, ${ }^{23}$ which corresponds to $\geq 2$ based on Sheard's criterion ${ }^{7,23}$ when converted into a continuous variable, which implies that Sheard's criterion is not met when values are lower than 2 .

\section{Ethical considerations}

Written informed consents for access to the schools were obtained from the Department of Education and the school principals, and consents and assents were obtained from parents and children, respectively. The study protocol was approved by the Biomedical Research Ethics Committee (ethical clearance reference number: BE 177/12) of the University of KwaZulu-Natal, South Africa. The conduct of the study complied with the Declaration of Helsinki regarding research on human subjects. ${ }^{47}$

\section{Results}

\section{Sample characteristics}

A total of 1211 individuals returned their consent and assent forms, but 10 students were excluded (7 had amblyopia, 1 was diabetic, 1 had glaucoma and another had corneal scars because of trauma), leaving 1201. A further 145 children aged 19 and above were excluded from this study, as we intended to report only on participants of the official high school age 
TABLE 2: Descriptive statistics for near heterophorias and fusional vergences.

\begin{tabular}{|c|c|c|c|c|c|c|c|c|c|c|c|}
\hline \multirow[t]{3}{*}{ Parameter } & \multicolumn{3}{|c|}{ Refractive errors (D) } & \multicolumn{4}{|c|}{ Heterophoria (pd) } & \multicolumn{4}{|c|}{ Near-fusional vergences (pd) } \\
\hline & \multirow[t]{2}{*}{ Myopia } & \multirow[t]{2}{*}{ Hyperopia } & \multirow[t]{2}{*}{ Astigmatism } & \multicolumn{2}{|c|}{ Distance } & \multicolumn{2}{|c|}{ Near } & \multicolumn{2}{|c|}{ Negative } & \multicolumn{2}{|c|}{ Positive } \\
\hline & & & & Lateral & Vertical & Lateral & Vertical & Break & Recovery & Break & Recovery \\
\hline Mean & -1.00 & 0.50 & -1.00 & -0.09 & 0.026 & -1.78 & 0.03 & 17.35 & 12.51 & 25.38 & 17.50 \\
\hline SD & 0.80 & 0.20 & 0.82 & 1.71 & 0.290 & 4.24 & 0.36 & 5.44 & 4.23 & 9.15 & 6.77 \\
\hline Median & -0.70 & 0.50 & -1.00 & 0.00 & 0.000 & 1.00 & 0.00 & 18.00 & 12.00 & 25.00 & 18.00 \\
\hline Min & -6.00 & 0.00 & -4.50 & -12.00 & -0.500 & -15 & -2.00 & 2.00 & 0.00 & 2.00 & 0.00 \\
\hline Max & -1.00 & 2.0 & 0.00 & 18.00 & 8.000 & 18.00 & 8.00 & 45.00 & 35.00 & 45.00 & 40.00 \\
\hline Range & 5.00 & 1.00 & 4.50 & 30.00 & 8.500 & 33.00 & 10.00 & 43.00 & 35.00 & 43.00 & 40.00 \\
\hline 1st quart & -1.00 & 1.00 & 0.00 & -1.00 & 0.000 & 1.00 & 0.00 & 14.00 & 10.00 & 20.00 & 14.00 \\
\hline 3rd quart & -1.00 & 1.00 & -0.75 & 0.00 & 0.000 & 9.00 & 0.00 & 20.00 & 16.00 & 30.00 & 20.00 \\
\hline Skew & -2.91 & 5.00 & -2.90 & 0.67 & 21.270 & 0.26 & 14.91 & 0.66 & -0.06 & 0.09 & 0.35 \\
\hline Kurtosis & 10.20 & 26.50 & 10.90 & 26.76 & 530.240 & 2.16 & 279.68 & 2.05 & 1.46 & -0.41 & 1.47 \\
\hline
\end{tabular}

Note: For phorias, minus signs for the mean indicate exophoria; positive signs indicate esophoria.

$n=1056$ participants aged $13-18$ years.

D, dioptres; pd, prism dioptres.

1st quart, 1st quartile; 3rd quart, 3rd quartile; SD, standard deviation

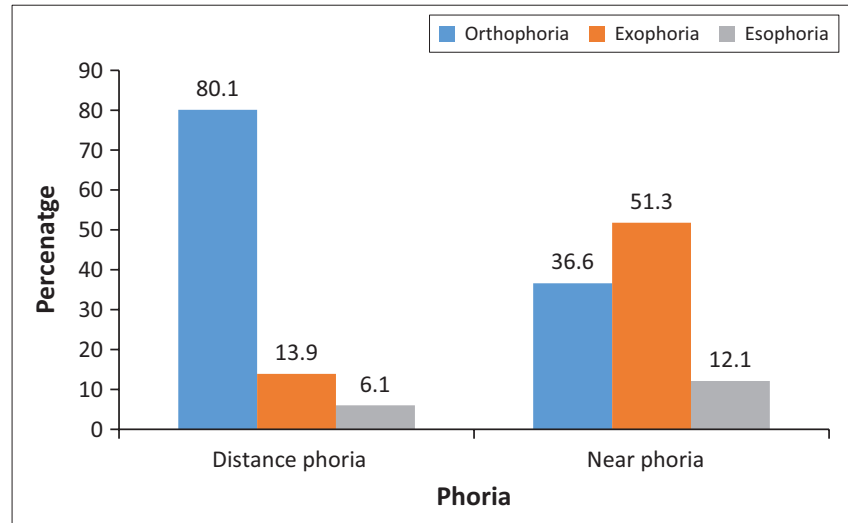

FIGURE 1: Prevalence of heterophorias at distance and near.

range of 13-18 years. Thus, data were analysed for 1056 participants (a response rate of $87 \%$ ), with a mean age of $15.89 \pm 1.58$ years and a median age of 16 years. There were $403(38 \%)$ males and $653(62 \%)$ females. The sample comprised $781(73.96 \%)$ students from grades 8-10 (lower grade level) and 275 (26.07\%) from grades 11-12 (higher grade level).

There was a correlation between the mean spherical equivalents of the left and right eyes $(r=0.645, p=0.01)$. The descriptive statistics for refractive errors, heterophoria and fusional vergences are shown in Table 2. Overall, data for refractive errors and phoria were not normally distributed, whereas data for fusional vergences were.

\section{Frequency distribution of phoria at distance fixation $(6.00 \mathrm{~m})$ and near fixation $(0.40 \mathrm{~m})$}

The frequencies of distance phoria are presented (Figure 1) with $95 \%$ confidence intervals (CI) in text. For distance lateral phoria, orthophoria was the most frequent at $80.1 \%$ (CI: $77.7 \%$ - 82.6\%); exophoria was $13.9 \%$ (CI: $11.9 \%-$ $16.0 \%)$, with the majority being $1 \mathrm{pd}$; while esophoria was $6.0 \%$ (CI: $4.5 \%-7.4 \%$ ), with the majority being $1 \mathrm{pd}$.

For lateral phoria at near (Figure 1), exophoria was the most frequent $(51.30 \%$; CI: $48.3 \%-54.2 \%)$. The majority were between 2 and 3 pd, while esophoria was 12.1\% (CI: 10.2\% $14.0 \%$ ) and mainly $2 \mathrm{pd}$.
For vertical phoria, hyperphoria at distance was $2.7 \%$ and $3.6 \%$ at near, while orthophoria was $97.3 \%$ and $96.4 \%$, respectively.

For the distribution of phorias according to demographic variables, the male participants were significantly more exophoric (mean $2.88 \pm 3.66 \mathrm{pd}$, median $2 \mathrm{pd}$, frequency $57 \%$ ) than females (mean $2.25 \pm 3.22 \mathrm{pd}$, median $2 \mathrm{pd}$, frequency $48.8 \%)(\mathrm{OR}, 2.73 ; p=0.01$, logistic regression). Neither age $(p=0.48)$ nor grade level $(p=0.64)$ influenced the distribution of exophoria. The prevalence of near esophoria was significantly higher among children aged 13 years compared to others aged $14-18$ years $\left(\chi^{2}=18.884\right.$, $p=0.02$; mean value $=F=2.382, d f=1047, p=0.03)$ and school grade $\left(\chi^{2}=6.578, p=0.01\right)$. There was no gender difference in the mean distance lateral phoria $(p=0.09)$, while mean distance vertical phoria was higher in older age group $(p=0.02)$.

For the distribution of near heterophoria and fusional vergences, the mild categories of exophoria and PFV and normal NFV were most prevalent (Table 3).

\section{Correlations between near heterophoria and near fusional vergence ranges}

The associations between fusional vergences and heterophoria were specific to low magnitudes of fusional vergences (Table 4).

\section{Exophoria and fusional vergences}

There was an inverse correlation between near exophoria and PFV break $(r=-0.095, p=0.01)$ and recovery $(r=-0.138$, $p=0.01$, whereas there was a positive correlation between exophoria and NFV $(r=0.148, p=0.01)$. The PFV break and recovery values were reduced (poorer) with an increase in the severity of the heterophoria. However, the association was significant only for mild PFV $(F=2.639, d f=928, p=$ 0.04). Participants with severe exophoria had significantly worse PFV values than did those in the mild and moderate categories (Table 4). 


\section{Esophoria and fusional vergences}

Esophoria was inversely correlated with NFV break values $(r=-0.136, p=0.01)$ and recovery $(r=0.136, p=0.01)$. For both severity of esophoria ( $>2$ and $\leq 2 \mathrm{pd}$ ), an increase in the magnitude was associated with poorer (lower) NFV values. The difference was significant only for anomalous NFV break $(p=0.01)$. A similar trend was observed for NFV recovery.

TABLE 3: Distribution of near heterophoria and near-fusional vergences for 1056 school children aged $13-18$ years.

\begin{tabular}{|c|c|c|c|c|}
\hline \multirow[t]{2}{*}{ Clinical measures } & \multirow[t]{2}{*}{ Frequency } & \multirow[t]{2}{*}{ Percentage } & \multicolumn{2}{|c|}{$95 \% \mathrm{Cl}$} \\
\hline & & & Lower limit & Upper limit \\
\hline \multicolumn{5}{|l|}{ Heterophoria (pd) } \\
\hline Orthophoria & 387 & 36.1 & 45 & 50.9 \\
\hline Exophoria & - & - & - & - \\
\hline Mild (1-7) & 432 & 41 & 38.3 & 44.1 \\
\hline Moderate (8-13) & 102 & 9.7 & 7.8 & 11.5 \\
\hline Severe $(>13)$ & 12 & 1.1 & 0.6 & 1.8 \\
\hline Total exophoria & 546 & 51.8 & & \\
\hline Esophoria & - & - & - & - \\
\hline$>2$ & 98 & 9.4 & 7.5 & 11.3 \\
\hline $1-2$ & 25 & 2.7 & 2.6 & 2.9 \\
\hline Total esophoria & 123 & 12.1 & 10.12 & 13.1 \\
\hline \multicolumn{5}{|c|}{ Fusional vergence breaks (pd) } \\
\hline Positive & - & - & - & - \\
\hline Mild (15-22) & 932 & 88.3 & 86.4 & 90.4 \\
\hline Moderate $(>7$ to $<15)$ & 107 & 10.1 & 8.2 & 11.9 \\
\hline Severe $(\leq 7)$ & 17 & 1.6 & 0.9 & 2.4 \\
\hline Negative & - & - & - & - \\
\hline Anomalous ( $\geq 22$ ) & 149 & 14.2 & 12.3 & 16.3 \\
\hline Normal $(<22)$ & 903 & 85.8 & 83.7 & 87.7 \\
\hline
\end{tabular}

$\mathrm{Cl}$, confidence interval; pd, prism dioptres.
With PFV, esophoria was positively correlated with PFV break $(r=0.132, p=0.03)$ and PFV recovery $(r=0.07, p=0.02)$. Positive fusional vergence values were higher with an increase in the magnitude of the esodeviation, and the association was significant only for mild PFV. A similar trend was observed for PFV recovery.

\section{Orthophoria and fusional vergences}

There was no significant correlation between orthophoria and PFV break $(r=-0.028, p=0.36)$, but there was negative correlation with recovery $(r=-0.07, p=0.02)$ and highly positive correlation with NFV break $(r=0.134, p=0.01)$ and recovery $(r=0.143, p=0.01)$.

\section{Vertical phoria and fusional vergence}

There was no significant correlation between hyperphoria and PFV values (break $=r=-0.031, p=0.85$; recovery: $r=-0.03, p=0.82)$, whereas hyperphoria $>0.5 \mathrm{pd}$ was inversely correlated with NFV $(r=-0.31, p=0.05)$. The mean NFV break values were significantly poorer (lower) with an increase in the severity of the hyperdeviation. However, the difference was significant only for anomalous NFV break values. A similar trend was observed for NFV recovery $(t=5.37, d f=1044, p=0.01)$

\section{Sheard's criterion and fusional convergence ratio}

A total of $6.45 \%$ of the children with exophoria failed the Sheard's criterion. There was no significant gender

TABLE 4: Associations between heterophorias and fusional vergences.

\begin{tabular}{|c|c|c|c|c|c|}
\hline \multirow[t]{2}{*}{ Heterophoria (pd) } & \multicolumn{3}{|c|}{ Positive fusional vergence break (pd) } & \multicolumn{2}{|c|}{ Negative fusional vergence break (pd) } \\
\hline & Mild & Moderate & Severe & Anomalous & Normal \\
\hline Orthophoria (Mean/SD) & $26.99 \pm 7.48$ & $10.97 \pm 2.47$ & $5.00 \pm 1.06$ & $15.24 \pm 3.87$ & $27.59 \pm 3.80$ \\
\hline \multicolumn{6}{|l|}{ Exophoria } \\
\hline Mild (Mean/SD) & $28.18 \pm 7.8$ & $11.28 \pm 2.22$ & 4.401 .67 & $16.17 \pm 3.44$ & $27.60 \pm 0.03$ \\
\hline Moderate (Mean/SD) & $26.07 \pm 7.38$ & $10.65 \pm 2.0$ & $6.00 \pm 1.32$ & $16.72 \pm 2.67$ & $25.24 \pm 1.09$ \\
\hline Severe (Mean/SD) & $24.75 \pm 9.21$ & $10.67 \pm 1.03$ & $5.00 \pm 1.40$ & $13.56 \pm 4.97$ & $25.00 \pm 0.00$ \\
\hline \multicolumn{6}{|l|}{ Stat test } \\
\hline$F$ & 2.63 & 0.51 & 0.76 & - & - \\
\hline$d f$ & 928 & 106 & 16 & - & - \\
\hline$p$ & 0.04 & 0.67 & 0.53 & 0.12 & 0.01 \\
\hline \multicolumn{6}{|l|}{ Esophoria } \\
\hline Esophoria $\leq 2$ (Mean/SD) & $27.26 \pm 7.74$ & $10.96 \pm 2.15$ & $4.80 \pm 1.26$ & $16.04 \pm 3.42,16.00$ & $27.21 \pm 3.83$ \\
\hline Esophoria > 2 (Mean/SD) & $28.72 \pm 7.09$ & $11.14 \pm 3.02$ & $6.00 \pm 1.6$ & $12.75 \pm 4.61,13.00$ & $27.19 \pm 3.14$ \\
\hline \multicolumn{6}{|l|}{ Stat test } \\
\hline$d f$ & 1046 & 6.434 & 14 & 107 & - \\
\hline$p$ & 0.03 & 0.88 & 0.37 & 0.01 & 0.92 \\
\hline \multicolumn{6}{|l|}{ Hyperphoria } \\
\hline Hyper $\leq 0.50$ (Mean/SD) & $27.34 \pm 7.60$ & $10.98 \pm 2.21$ & $4.93 \pm 1.28$ & $15.83 \pm 3.58,16.00$ & $27.22 \pm 3.75$ \\
\hline Hyper > 0.50 (Mean/SD) & $29.03 \pm 9.47$ & $10.80 \pm 2.28$ & $5.00 \pm 1.41$ & $13.24 \pm 4.82,14.00$ & - \\
\hline \multicolumn{6}{|l|}{ Stat test } \\
\hline$t$ & -0.98 & 0.178 & -0.069 & - & - \\
\hline$d f$ & 31.35 & 105 & 15 & - & - \\
\hline$p$ & 0.33 & 0.85 & 0.94 & 0.03 & - \\
\hline
\end{tabular}

Note: Bold values indicate variables with significant association.

$n=1056$ schoolchildren aged $13-18$ years.

SD, standard deviation; pd, prism dioptres. 
$\left(\chi^{2}=1.96, d f=1, p=0.16\right)$, age group $\left(\chi^{2}=2.176, d f=1, p=0.14\right)$ or grade level $\left(\chi^{2}=0.79, d f=1, p=0.37\right)$ difference in the failure of Sheard's criteria. The mean fusional convergence ratio was 4.67 , with a median of 1.16 . The Mann-Whitney test showed no significant difference in fusional convergence ratio in gender $(p=0.15)$ and age $(p=0.98)$.

\section{Associations between near heterophoria and refractive errors}

Exophoria was significantly associated with astigmatism $\left(\chi^{2}=11.1, d f=3, p=0.01\right)$; while esophoria was significantly associated with myopia $\left(\chi^{2}=25.7, d f=1, p=0.01\right)$, astigmatism $\left(\chi^{2}=34.2, d f=1, p=0.01\right)$ and anisometropia $\left(\chi^{2}=38.7, d f=1, p=0.01\right)$.

\section{Discussion}

The distribution of heterophoria and its correlation with fusional vergences and refractive errors were investigated in a sample of schoolchildren aged 13-18 years. Heterophoria was not normally distributed, and orthophoria and exophoria were most prevalent at distance and near fixations, respectively. Participants with exophoria had significantly reduced PFV ranges and increased NFV ranges, while those with esophoria had significantly reduced NFV ranges but increased PFV ranges. There was a weak but significant correlation between phoria and fusional vergences, which may be related to the associations being specific to the mild severities or magnitudes. These findings suggest that decompensation and the corresponding manifestation of symptoms are likely to occur at the mild levels of phoria and fusional vergences.

The preponderance of orthophoria at distance agrees with findings from most studies, ${ }^{4,8,10,14,15,16,17}$ but contrasts with those of Akpe et al., ${ }^{12}$ who found esophoria to be more prevalent at distance than exophoria. The mean distance phoria tends to remain the same, that is, close to orthophoria throughout life. ${ }^{18}$ Orthophorisation, which is a tendency for a high incidence of orthophoria at distance in most populations, may be because of vergence adaptation. ${ }^{18}$ At near fixation, the preponderance of exophoria is similar to findings from other studies, ${ }^{8,16,23,24}$ but contrasts with others ${ }^{4,20}$ which found a higher prevalence of orthophoria at near. The mean value of lateral phoria in the present study is comparable to findings reported in several studies, ${ }^{8,19,23,24,31}$ lower than findings from some studies ${ }^{11,16,22}$ and higher than others. ${ }^{4,15,21,25,26}$ It is known that the mean phoria at near seems, to remain fairly stable with age, within approximately $2 \mathrm{pd}$ exophoria for the majority of children. ${ }^{4}$ The differences in mean phoria reported across studies are influenced by several methodological variables, which include age and study methods.

Vergence measures have been suggested to vary with race, ethnicity and age. ${ }^{19}$ Specifically for race, the mean findings of heterophoria in the present study are comparable to those from other African populations ${ }^{10,13}$ (Table 5) $11,10,12,31,8,24$, $42,26,14,27,23,21,4,22,15,25,16,20,19$; the slight differences may be because of those studies ${ }^{11,13}$ that enrolled younger schoolchildren. Furthermore, different phoria dissociation methods were used in some studies. ${ }^{10,13}$ Akpe et al. ${ }^{13}$ used Maddox Rod and Maddox Wing tests and Mathebula used the Maddox rod, whereas the present study used the von Graefe technique. Comparing the prevalence of phoria found in this study to other studies on African populations, the $51.8 \%$ prevalence of exophoria at near is lower than that of $86.7 \%$, and reported by Ovenseri-Ogbomo and Assien. ${ }^{9}$ The $12 \%$ prevalence of near esophoria is higher than the $10.8 \%$ reported by Akpe et al. ${ }^{13}$ and $2 \%$ by Ovenseri-Ogbomo. ${ }^{19}$

TABLE 5: Summary of findings on means and standard deviations for lateral heterophoria at far and near fixations.

\begin{tabular}{|c|c|c|c|c|c|c|c|}
\hline Author(s) & Country of study & Study setting & Age (years) & Sample size & Technique & Far horizontal (pd) & Near horizontal (pd) \\
\hline Present study & South Africa & SS & $13-18$ & 1056 & Von Graefe & $-0.1 \pm 1.71$ & $-1.7 \pm 4.21$ \\
\hline Mathebula $^{11}$ & South Africa & CS & $20-36$ & 139 & Von Graefe & N/A & $-2.1 \pm 6.20$ \\
\hline Mathebula $^{10}$ & South Africa & SS & $6-13$ & 900 & Maddox rod & $-0.2 \pm 1.17$ & $-2.5 \pm 2.37$ \\
\hline Makgaba $^{12}$ & South Africa & CS & $18-38$ & 336 & Von Graefe & $-0.7 \pm 2.87$ & $-3.8 \pm 4.80$ \\
\hline Razavi et al. ${ }^{31}$ & Iran & CS & $20-40 ; M / A=25 \pm 3.6$ & 111 & Maddox wing & N/A & $-2.2 \pm 3.00$ \\
\hline Zwierko et al. ${ }^{8}$ & Poland & - & $M / A=21 \pm 0.67$ & 24 & Maddox wing & $0 \pm 0$ & $-2.3 \pm 0.57$ \\
\hline Radocivc et al. ${ }^{24}$ & Poland & SS & $6-7$ & 152 & Cover test & $0.1 \pm 1.5$ & $-2.3 \pm 3.80$ \\
\hline Myklebust $^{42}$ & Norway & SS & $5-11$ & 90 & Cover test & $0.3 \pm 2.8$ & $2.5 \pm 3.91$ \\
\hline Jimenez et al. ${ }^{26}$ & Spain & SS & $6-12$ & 1056 & Modified Thorington & $+0.7 \pm 4.4$ plus (eso) & $-0.1 \pm 3.80$ \\
\hline Aring et al. ${ }^{14}$ & Sweden & SS & $4-15$ & 143 & Cover test & $\mathrm{N} / \mathrm{A}$ & -4 pd for exo \\
\hline Abdi et al. ${ }^{27}$ & Sweden & SS & $6-16$ & 216 & Cover test & $\mathrm{N} / \mathrm{A}$ & N/A \\
\hline Lanca and Rowe ${ }^{23}$ & Portugal & SS & $6-14$ & 530 & Cover test & $-0.1 \pm 0.66$ & $-1.8 \pm 2.63$ \\
\hline Lyon et al. ${ }^{21}$ & USA & SS & $6-11$ & 879 & Modified Thorington & 0.00 & $-1 \pm 4.00$ \\
\hline Walline et al. ${ }^{4}$ & USA & SS & Grades 2, 5 & 1495 & Cover test & $-0.02 \pm 0.51$ & $-0.2 \pm 1.59$ \\
\hline Jackson and Goss ${ }^{22}$ & USA & CS & $7.9-15.9$ & 244 & Von Graefe & $-1 \pm 2$ & $-3.0 \pm 4.00$ \\
\hline Letourneau \& Giroux ${ }^{15}$ & Canada & SS & $6-13$ & 2035 & Maddox & $+0.5 \pm 2.54$ & $-0.7 \pm 4.51$ \\
\hline Junghans et al. ${ }^{25}$ & Australia & SS & $3-12$ & 2697 & Cover test & $-0.1 \pm 1.58$ & $-1.0 \pm 2.53$ \\
\hline Leone et al. ${ }^{16}$ & Australia & SS & $6-12$ & 4093 & Cover test & N/A & 12 -year-olds $=-3.9$ \\
\hline Chen et al. ${ }^{20}$ & Malaysia & SS & $2-15$ & 268 & Maddox wing & N/A & -1.39 \\
\hline Chen and Abidin $^{19}$ & Malaysia & SS & $7-12$ & 60 & Howard Card & $-0.2 \pm 0.82$ & $-1.8 \pm 3.90$ \\
\hline
\end{tabular}

Note: More than one reference used in table. Exophoria values are represented by minus signs, whereas esophoria values are indicated by plus signs. $M / A$, mean age; $C S$, clinic setting; SS, school setting; N/A, not applicable.

pd, prism dioptres. 
For non-African populations, the $51.8 \%$ prevalence of exophoria at near is similar to the finding of $52.2 \%$ by Leone et al. ${ }^{16}$ and higher than that of $19.7 \%$ by Chen et al. ${ }^{20}$ The $12 \%$ prevalence of near esophoria is higher than the finding of $10.4 \%$ by Leone et $a .^{16}$ and lower than that of $16.1 \%$ by Chen et al. ${ }^{20}$ The prevalence of vertical phoria is generally low. The $3.6 \%$ found in the present study is the same as that of Razavi et al. ${ }^{31}$ Junghans et al. ${ }^{25}$ found vertical phoria (>0.5 pd) of $0.5 \%$, Leone et al. ${ }^{16}$ found $0.2 \%$ and Akpe et al. ${ }^{13}$ found no vertical phoria. Although vertical phorias are rarely reported and have a low prevalence, they are sometimes clinically important, causing diplopia and degrading the perception of depth. ${ }^{11}$ The differences in study methods influences the prevalence of the measures; therefore, the means may be a more reliable way of comparing variables.

Relating to demographics, most studies found no difference in the prevalence of phorias according to gender or age. ${ }^{4,16,20,24,31}$ In the present study, males were significantly more exophoric than females, in agreement with Akpe et al. ${ }^{13}$ In the present study, phoria was not significantly associated with age, which agrees with findings from most studies. ${ }^{15,20,22,26,27,32}$ Comparing visual parameters with age depends largely on the participants' age range and intervals; and, in most cases, longitudinal studies are a better study design to make better inferences about such associations. The mean near phoria becomes more exophoric with increasing age..$^{4,16,26,28,29}$ Studies with adults were highlighted (Table 5) to enable a comparison of phoria trends beyond the age of 18 years. Regardless of the diverse study designs, the data from various studies (Table 5) reveals a lack of marked differences in the distribution of mean values of near phoria up to the age of 40 years, with a maximum of 4 pd exophoria reported at near. A trend in the distribution of phoria across the ages shows that beyond 6 years of age, when a child first begins school, a greater incidence of heterophoria may occur because of changes in the visual system that may be related to the start of school activities. ${ }^{20,26,48}$ The development of near exophoria is an adaptive mechanism to near-point stress, beyond which there could be decompensation. ${ }^{48}$ From the age of 20, humans become progressively more exophoric for near vision, ${ }^{4,30}$ reaching around $5 \mathrm{pd}$ at the age of $50 .{ }^{4}$ In general, differences in findings across studies are influenced by factors, which include the technique used for dissociation, level of illumination, accommodative stimuli and examiner factors. ${ }^{1,10}$

\section{Near phoria, fusional vergences and refractive errors}

There was a weak but significant correlation between phoria and fusional vergences, which may be related to their associations being specific to categories, for example, mild PFV. Similar to the present study, most studies reported weak associations between heterophoria and fusional vergences, ${ }^{23,24,32}$ while others found no correlation. ${ }^{30,31,35}$ Anderson et al..$^{28}$ found significant associations, although the extent of the associations were not quantified. Associations between phoria and fusional vergence are expected, given that heterophoria is compensated by fusional vergences, which vary with the direction of the phoria. ${ }^{34,35}$ Accordingly, exophoria exists because of a partial failure of base-out prism adaptation, while esophoria is because of a partial failure to adapt to base-in prism. ${ }^{17,18}$

The association between phorias and fusional vergences becomes clinically important in cases of decompensation. Thus, a knowledge of the magnitudes or severities of the vergence reserves and demand give useful clues as to the possibility of having comfortable binocular vision. Invariably, the report of symptoms is important in optometric practice, as most patients who consult do so because of the symptoms they are experiencing and an analysis of patients' symptoms guides a clinician's treatment plans. Aligned to heterophoria and fusional vergences is Sheard's criterion, which is a good indicator of symptoms. ${ }^{46}$ The $6.5 \%$ failure rate of Sheard's criterion in this study is lower than the $12.5 \%$ found by Myklebust et al. ${ }^{42}$ in Norwegian schoolchildren, while the 4.6 mean convergence ratio found is comparable to $4.45 \mathrm{pd}$ by Lanca and Rowe in Portugal. ${ }^{23}$

The findings on the association of phorias and refractive errors from previous studies are diverse, ${ }^{37,38,39,40,41,42}$ which may be related to different study designs and criteria applied to define variables. Studies of the association between phoria and refractive errors of African schoolchildren could not be found. However, other studies found associations between near phoria and refractive errors. ${ }^{13,16,36,38}$ In the present study, esophoria was associated with myopia, which agrees with the findings by Chung and Chong ${ }^{36}$ but contrasts with those of Ditmars, ${ }^{37}$ where myopic patients tended to be exophoric, whereas hyperopes tended to be esophoric. Li-Ju et al. ${ }^{38}$ also found a significant association between exophoria and myopia in Taiwanese schoolchildren. Similar to the present study, Walline et al. $^{4}$ reported no association between hyperopia and esophoria. The absence of significant associations between near esophoria and hyperopia may be related to the mainly low magnitudes of hyperopia found in the present study.

In general, the association between heterophorias and refractive errors may be considered from different perspectives. In esophoria resulting from high latent hyperopia, the esophoria may be such that the motor and sensory fusion cannot cope, resulting in decompensation and tropia. ${ }^{39}$ The association may be because of the need for greater accommodative efforts to overcome hyperopia, which in conjunction with convergence causes an esodeviation. ${ }^{16}$ Furthermore, near esophoria is associated with the development and increased rates of myopic progression in children ${ }^{33,36,40}$ and is related to vergence adaptation to a prolonged exposure to near tasks, which causes a convergence; this may even be a possible cause of myopia progression. ${ }^{33,34}$ 


\section{Implications, applications and limitations of study findings}

In summary, this study extends our understanding and knowledge of the distribution of heterophoria and its associations with fusional vergences and refractive errors in a sample of South African schoolchildren. Given that heterophoria is symptomatic only when it is decompensated, the significant correlations with fusional vergences, as well as the percentage of children who failed Sheard's criterion, deserve attention, as the failure of Sheard's criterion is regarded as a good indicator of symptoms. ${ }^{46}$ The participants in the study may not have reported symptoms at the time of vision screening. However, with changes in phoria associated with increase in age and near demands, the subsequent decrease in PFV values may result in phoria being decompensated. This is considerable, especially as such a compromise can occur with severe phoria and lower levels of fusional vergences. This study provides epidemiological data on heterophoria and fusional vergence for high school children. The findings reported can guide clinicians and researchers on the expected magnitudes of heterophorias and fusional vergences where phoria is likely to become decompensated under increased near tasks.

The strengths of this study include the random sampling employed, the use of many schools with a relatively large sample size and the collection of data by only one examiner, suggesting that the findings from this study may be considered representative and could be extrapolated to the entire population of black African schoolchildren in the municipality. However, there are some limitations that may influence the interpretation of the findings. For instance, although the von Graefe technique is a commonly used phoria measurement technique, a limitation is that it may give relatively higher exophoria values. ${ }^{49}$ The use of this technique to assess heterophoria and fusional vergence would have allowed for consistency in technique. The intention in the present study was to apply techniques as close as possible to those used in the convergence insufficiency projects ${ }^{50}$ that formed the basis of the present study. However, other authors ${ }^{51,52}$ used the von Graefe technique (for phoria) and the trial frame and prism bar (for fusional reserves), as in this study, and this allowed for comparison. The prism bar on a trial frame is a preferred tool in the screening setting as it is quicker, allows the view of eye movement and makes it easier for school-aged children to follow instructions. ${ }^{43}$ Furthermore, even when the Risley prism is used to measure fusional vergences, the prism power must still be set to zero while the examiner notes the appropriate compensating prism direction in mind, before the vergence measurements are commenced. That approach may be compared to starting the fusional measurement with a zero diopter prism. Another concern is the difference in the field of view between phoropter and prism bar, but it is unknown whether that could greatly influence findings. It might also be argued that other tests, such as vergence facility testing, negative relative accommodation, binocular accommodative facility with plus lenses and near point of convergence tests that also measure vergence functions, apply different testing techniques yet give gross information of the vergence functions.

\section{Conclusion}

The heterophoria results are comparable with those from other studies in South African populations. The correlation between phorias and fusional vergence were weak but significant and specific to the mild categories of phoria and fusional vergences.

\section{Acknowledgements}

I am grateful to Drs Rekha Hansraj and Percy Mashige for reading drafts of the manuscript.

\section{Competing interests}

The author declares that he has no financial or personal relationships that may have inappropriately influenced him in writing this article.

\section{References}

1. Von Noorden G, Campos E. Binocular vision and ocular motility: Theory and Management of Strabismus. 6th ed. St Louis, MO: Mosby; 2002.

2. Grosvenor T. Primary care optometry. 5th ed. Philadelphia, PA: Butterworth Heinemann Elsevier; 2007.

3. Babinsky E, Sreenivasan V, Candy TR. Near heterophoria in early childhood Invest Ophthalmol Vis Sci. 2015;56:1406-1415. https://doi.org/10.1167/ iovs.14-14649

4. Walline JJ, Mutti DO, Zadnik K. Development of phoria in children. Optom Vis Sci. 1998;75:605-610. https://doi.org/10.1097/00006324-199808000-00026

5. Saladin JJ. Effects of heterophoria on stereopsis. Optom Vis Sci. 1995;72:487-492. https://doi.org/10.1097/00006324-199507000-00008

6. Scheiman M, Wick B. Clinical management of binocular vision: Heterophoric, accommodative and eye movement disorders. 3rd ed. Philadelphia, PA: JB Lippincott; 2014.

7. Goss DA. Ocular accommodation, convergence, and fixation disparity: A manual of clinical analysis. Boston, MA: Butterworth-Heinemann; 1995.

8. ZwierkoT, Puchalska-Niedbał L, Krzepota J, Mikołaj M, Woźniak J. The effects of sports vision training on binocular vision function in female university athletes. J Human Kinetics. 2015;49:287-296.

9. Ovenseri-Ogbomo G, Assien R. Refractive error in school children in Agona, Ghana. S Afri Optom. 2010;69:86-92. https://doi.org/10.4102/aveh. v69i2.129

10. Mathebula SD, Sheni DDD, Oduntan AO. Distribution of heterophoria among primary school children of South Africa. S Afr Optom. 2002;61:48-54.

11. Mathebula SD. Investigations of the clinical relationships between accommodation and convergence tests. S Afr Optom. 2003;62:21-27.

12. Makgaba NT. A retrospective analysis of heterophoria values in a clinical population aged 18 to 30 years. S Afr Optom. 2006:65;150-156. https://doi. org/10.4102/aveh.v65i4.270

13. Akpe BA, Dawodu OA, Abadom EG. Prevalence and pattern of strabismus in primary school pupils in Benin City, Nigeria. Nigerian J Ophthalmol. 2014;22:38-43. https://doi.org/10.4103/0189-9171.142755

14. Aring E, Gronlund MA, Andersson S. Strabismus and binocular functions in a sample of Swedish children aged 4-15 years. Strabismus. 2005;13:55-61. https:// doi.org/10.1080/09273970590922664

15. Letourneau JE, Giroux R. Non-Gaussian distribution curve of heterophoria in children. Optom Vis Sci. 1991;68:132-137. https://doi.org/10.1097/00006324199102000-00008

16. Leone JF, Cornell E, Morgan IG, et al. Prevalence of heterophoria and associations with refractive error, heterotropia and ethnicity in Australian school children. Br J Ophthalmol. 2010;94:542-546. https://doi.org/10.1136/ bjo.2009.163709

17. Dowley D. Heterophoria. Optom Vis Sci. 1990;67:456-460. https://doi.org/ 10.1097/00006324-199006000-00010 
18. Dowley D. The orthophorization of heterophoria. Ophthal Physiol Opt. 1987;7:169-174. https://doi.org/10.1111/j.1475-1313.1987.tb01016.x

19. Chen AH, Abidin AHZ. Vergence and accommodation systems in Malay primary school children. J Biomed Sci. 2002;9:9-15.

20. Chen AH, O'Leary DJ, Howell E. Near visual function in children. Part I: Near poin of convergence. Part II: Amplitude of accommodation. Part III: Near heterophoria. Ophthal Physiol Opt. 2000;20:185-198. https://doi.org/10.1016/S0275-5408(99) 00056-3

21. Lyon WD, Goss D, Horner D, Downey J, Rainey, B. Normative data for modified Thorington phorias and prism bar vergences from the Benton-IU study. Optometry. 2005;76:593-599. https://doi.org/10.1016/j.optm.2005.08.014

22. Jackson TW, Goss DA. Variation and correlation of standard clinical phoropter tests of phorias, vergence ranges, and relative accommodation in a sample of school-age children. J Am Optom Assoc. 1991;62:540-547.

23. Lança C, Rowe FJ. Variability of fusion vergence measurements in heterophoria. Strabismus. 2016;24:63-69. https://doi.org/10.3109/09273972.2016.1159234

24. Radaković M, Ivetić V, Naumović N, Čanadanović V, Stankov B. Heterophoria and fusional convergence and divergence in pre-school children. Medicinski Glasnik. 2012;9:293-298.

25. Junghans B, Keily $P$, Crewther DP, Crewther SG. Referral rates for a functional vision screening among a large cosmopolitan sample of Australian children vision screening among a large cosmopolitan sample of Australian children.
Ophthalmic Physiol Opt. 2002; 22:10-25. https://doi.org/10.1046/j.1475-1313. Ophthalmic Phys
2002.00010.x

26. Jiménez R, Pérez MA, García JA, González MD. Statistical normal values of visual parameters that characterize binocular function in children. Ophthal Physiol Opt. 2004;24:528-542. https://doi.org/10.1111/j.1475-1313.2004.00234.x

27. Abdi S, Lennerstrand G, Pansell T, Rydberg A. Orthoptic findings and asthenopia in a population of Swedish schoolchildren aged 6 to 16 years. Strabismus 2008; 16:47-55. https://doi.org/10.1080/09273970802020243

28. Anderson H, Stuebing KK, Fern KD, Manny RE. Ten-year changes in fusional vergence, phoria, and nearpoint of convergence in myopic children. Optom Vis Sci. 2011;88:1060-1065. https://doi.org/10.1097/OPX.0b013e31822171c0

29. Abraham NG, Srinivasan K, Thomas J. Normative data for near point of convergence, accommodation, and phoria. Oman J Ophthalmol. 2015;8:14-18. https://doi.org/10.4103/0974-620X.149856

30. Weymouth FW, Brust PR, Goba FH. Ocular muscle balance at the reading distance and certain related factors. Am J Physiol Opt. 1925;6:184-205.

31. Razavi M, Poor S, Daneshyar A. Normative values for the fusional amplitudes and the prevalence of heterophoria in adults. Iranian J Ophthalmol. 2010;22:41-46.

32. Scobee RG, Green EL. Relationships between lateral heterophoria, prism vergence and the near point of convergence. Am J Ophthalmol. 1948;31:427-441. https:// doi.org/10.1016/0002-9394(48)92164-3

33. Conway ML, Jennifer $\mathrm{T}$, Ahalya $\mathrm{S}$. Is the aligning prism measured with the Mallett unit correlated with fusional vergence reserves? PLoS One. 2012;7e42832.

34. Sreenivasan V, Irving E, Bobier W. Effect of heterophoria type and myopia on accommodative and vergence responses during sustained near activity in children. Vision Res. 2012;57:9-17. https://doi.org/10.1016/j.visres.2012.01.011
35. Rowe FJ. Fusional vergence measures and their significance in clinical assessment. Strabismus. 2010;18:48-57. https://doi.org/10.3109/09273971003758412

36. Chung KM, Chong EC. Near esophoria high is associated with myopia. Clin Exp Optom. 2000;83(2):71-75. https://doi.org/10.1111/j.1444-0938.2000.tb04895.x

37. Ditmars DL. Relationship between refractive error, phoria, and interpupillary distance in 500 patients. J Am Optom Assoc. 1966;37:361-363.

38. Li-Ju Lai, Wei-Hsiu H, Chien-Neng K, et al. The relationship between myopia and ocular alignment among rural adolescents. Open J Prev Med. 2014;4: 834-843. https://doi.org/10.4236/ojpm.2014.411094

39. Evans BJW. Optometric prescribing for decompensated heterophoria. Optom Pract. 2008;9:63-78.

40. Goss DA. Variables related to the rate of childhood myopia progression. Optom Vis Sci. 1990;67:631-636. https://doi.org/10.1097/00006324-199008000-00014

41. Goss DA, Zhai H. Clinical and laboratory investigations of the relationship of accommodation and convergence function with refractive error: A literature review. Doc Ophthalmol. 1994;86:349-380. https://doi.org/10.1007/BF01204595

42. Myklebust A, Riddell P. Fusional stamina: An alternative to Sheard's criterion Scand J Optom Vis Sci [serial online]. 2016 [cited 2017 May 12];9:1-7. http:// www.sjovs.org/index.php/SJOVS/article/view/85

43. Wajuihian SO, Hansraj R. Vergence anomalies in a sample of high school students in South Africa. J Optom. 2016;9:246-257. https://doi.org/10.1016/j.optom.2015. 10.006

44. Wajuihian SO, Hansraj R. Association of symptoms with refractive, accommodative and vergence anomalies in high school children in South Africa. Optom Vis Perf. and vergence

45. Bade A, Boas M, Gallaway M, Mitchell L, Scheiman M, Kulp MT. Relationship between clinical signs and symptoms of convergence insufficiency. Optom Vis Sci. 2013;90:988-995. https://doi.org/10.1097/OPX.0000000000000012

46. Sheedy JE, Saladin JJ. Association of symptoms with measures of oculomotor deficiencies. Am J Optom Physiol Opt. 1978;55:670-676. https://doi.org/ 10.1097/00006324-197810000-00002

47. Declaration of Helsinki [homepage on the Internet]. [cited 2017 May 10]. Available from: http://www.who.int/bulletin/archives

48. Birnbaum $\mathrm{MH}$. Optometric management of near point vision disorders. Boston MA: Butterworth-Heinemann; 1993.

49. Goss DA, Moyer BJ, Teske MC. A comparison of dissociated phoria test findings with von Graefe phorometry and modified Thorington testing. J Behav Optom. 2008;19:145-149.

50. Rouse MW, Borsting E, Deland PN. Reliability of binocular vision measurements used in the classification of convergence insufficiency. Optom Vis Sci. 2002;79:254326. https://doi.org/10.1097/00006324-200204000-00012

51. Marran LF, De Land PN, Nguyen AL. Accommodative insufficiency is the primary source of symptoms in children diagnosed with convergence insufficiency. Optom Vis Sci. 2006;83:E281-E289. https://doi.org/10.1097/01.opx.0000216097. Vis Sci.

52. Porcar E, Martinez-Palomera A. Prevalence of general binocular dysfunctions in a population of university students. Optom Vis Sci. 1997:74:111-113. https://doi. org/10.1097/00006324-199702000-00023 